\title{
THE NUMBER OF STRUCTURES OF FINITE RELATIONS
}

\author{
ROBERT L. DAVIS ${ }^{1}$
}

1. Preliminaries. Let $A$ be a dyadic relation defined on a finite set of $n$ elements. It is convenient to regard $A$ as an $n \times n$ matrix over the two-element Boolean algebra, so that $a_{i j}=1$ if and only if $i A j$. There is then an obvious correspondence of relational concepts $[1 ; 2]^{2}$ with matrix notions, interpreting conjunction as "coordinatewise" multiplication, relative product as matrix product, etc. In this paper, however, matrix notation and Boolean 1's and 0's are adopted merely for convenient reference.

If $A$ and $B$ are two relations defined over the set $N=\{1, \cdots, n\}$, then they are isomorphic just when there is a permutation $\pi$ of $N$ such that $A$ has the same matrix with respect to $N$ as $B$ with respect to $\pi(N)$ [cf. 3, *151.01]. Formally, for every relation $A$ defined on $N$ and every $\pi$ in $\mathfrak{S}_{n}$ (the symmetric group of $n$ letters), define a transformation $t_{\pi}$ of $A$ by

$$
t_{x}(A)=t_{x}\left[\left(a_{i j}\right)\right]=\left(a_{x^{-1}(i) x^{-1}(j)}\right),
$$

so that $t_{\pi}$ sends each row and each column of $A$ into its $\pi$-image:

Definition. Two relations $A, B$ defined on $N$ are isomorphic if and only if there is a permutation $\pi$ in $\mathfrak{S}_{n}$ such that $t_{\pi}(A)=B$.

The structure of a relation, as understood for instance by Russell [4, p. 61] and Carnap, is simply the class of all relations to which it is isomorphic (what Whitehead and Russell at first called the relationnumber $\left.\left[3,{ }^{*} 151.02\right]\right)$. Now Carnap pointed out in his latest book $[6$, p. 124] that it was not even known how many structures there are for a dyadic relation on a finite set, despite the fact that such knowledge "would be of importance not only for deductive and inductive logic but also for certain branches of science." It turns out that this problem, together both with its generalization to arbitrary $m$-adic relations and its restriction to certain special kinds of dyadic relations, can be solved in terms of elementary group theory and various simple combinatorial arguments.

The theory of $m$-adic relations, namely those which somehow relate $m$ objects at once, has never drawn the close analysis given the

Presented to the Society, September 4, 1952; received by the editors June 1, 1952.

1 This paper was written while I was working on a Ford Foundation project. I should like to thank Rudolf Carnap for his kind encouragement and his many suggestions concerning the logical context of the problem and also its literature. I am also grateful to R. M. Thrall for pointing out a short form of the main formula. 
dyadic theory by such authors as Whitehead and Russell, McKinsey, and Tarski. Nevertheless, it is straightforward to extend the concepts of isomorphism and structure to the general case. An $m$-adic relation on $N$ is given by an ordered set of $n^{m} 1$ 's and 0's:

$$
A=\left(a_{i_{1}} \ldots i_{m}\right)
$$

which may be taken as an " $m$-way matrix" or tensor [cf. 7]. In extending the definition of isomorphism it is notationally convenient to write the transformation in terms of the inverse permutation:

$$
t_{x-1}(A)=\left(a_{\pi\left(i_{1}\right) \cdots \pi\left(i_{m}\right)}\right) \text {. }
$$

With this understanding the definition provides a criterion for isomorphism valid for an arbitrary $m$-adic relation defined on a finite set. As before, the structure of such a relation is the class of all relations isomorphic to it.

From these materials, derivation of the number of structures of a relation proceeds by a counting method well known in group theory. If $\Re_{n}^{m}$ is the set of all $m$-adic relations on $n$ elements, the $t_{\pi}$ 's defined above form a group isomorphic to $\mathfrak{S}_{n}$ which "acts on" $\mathfrak{R}_{n}^{m}$ in an obvious way. The process is summarized in the following remarks [8, pp. 98-115].

(i) If $G$ is a group of permutations of any set $R, G$ partitions $R$ into orbits (sets of transitivity) by the relation: $x$ equivalent to $y$ if and only if there is a $\pi$ in $G$ such that $\pi(x)=y$.

(ii) When $G$ has order $g$, the number, $o$, of these orbits is

$$
o=\frac{1}{g} \sum_{\boldsymbol{r} \in G} f(\pi)
$$

where $f(\pi)$ is the number of elements of $R$ fixed under $\pi$.

(iii) If $\pi$ and $\phi$ are conjugate elements in $G$, then $f(\pi)=f(\phi)$.

(iv) Each conjugate class $[\pi]$ in $\mathscr{S}_{n}$ is completely determined by the cycle structure of any of its members. For $1 \leqq k \leqq n$, let $p_{k}$ be the number of $k$-cycles in the disjoint-cycle representation of any member of $[\pi]$. Then the number of elements in the conjugate class $[\pi]$ is [cf. 9, p. 9]

$$
c([\pi])=\frac{n !}{p_{1} ! 1^{p_{1}} \cdots p_{n} ! n^{p_{n}}} .
$$

Since the group of $t_{\pi}$ 's is isomorphic to $\mathfrak{S}_{n}$, and since the structure of a relation is just an orbit in $\Re_{n}^{m}$, the desired number of structures is

$$
s t_{m}(n)=\frac{1}{n !} \sum_{\pi \in \Theta_{n}} f\left(t_{\pi}\right)=\sum_{[\pi]} n(\pi) f\left(t_{\pi}\right),
$$


where $n(\pi)=(1 / n !) c([\pi])$ and the latter summation is over all conjugate classes $[\pi]$ in $\widetilde{S}_{n}$.

2. Counting lemma. The problem now is: Given a conjugate class in $\mathfrak{S}_{n}$, to find how many relations are fixed under one of its representatives. Once for all, let us agree always to choose one canonical representative from each class, written in a fixed canonical way: to wit, for the class with cycle structure $\left(p_{1}, \cdots, p_{n}\right)$, always choose the permutation with $p_{1} 1$-cycles $(1), \cdots,\left(p_{1}\right)$ written first, then $p_{2}$ 2 -cycles $\left(p_{1}+1, p_{1}+2\right), \cdots,\left(p_{1}+2 p_{2}-1, p_{1}+2 p_{2}\right)$, and so on. Thus, for instance, if the first nonzero cycle-number is $p_{h}, \pi$ will begin with $(12 \cdots h)$. An arbitrary $k$-cycle of $\pi$, not necessarily the first cycle, will be written $\left(1^{\prime} 2^{\prime} \cdots k^{\prime}\right)$ to indicate merely that it consists of some number and its $k-1$ successors in order.

Now suppose $\pi$ contains the cycles $(12 \cdots h)$ and $\left(1^{\prime} 2^{\prime} \cdots k^{\prime}\right)$ and suppose $t_{\pi}(A)=A$. Let $A_{0}$ be the submatrix consisting of the rows $1, \cdots, h$ of $A$ and the columns $1^{\prime}, \cdots, k^{\prime}$. The effect of $\pi$ on the submatrix $A_{0}$ is fully determined by the effect of $(12 \cdots h)$ on its rows, and that of $\left(1^{\prime} 2^{\prime} \cdots k^{\prime}\right)$ on its columns.

Hence, if $a_{i j^{\prime}}$ is any entry of $A_{0}$, the requirement that $t_{\pi}(A)=A$ establishes equations between $h k$ ostensibly distinct entries

$$
a_{i j^{\prime}}=a_{i+1, j^{\prime}+1}=a_{i+2, j^{\prime}+2}=\cdots=a_{i+h k, j^{\prime}+h k}
$$

in view of the choice of $\pi$. Since the row indices are computed modulo $h$ and the column indices modulo $k$, all of these entries actually are distinct only when $h$ and $k$ are relatively prime. In general, if their least common multiple is $c$, then the first $c$ entries are distinct, while the rest merely repeat them.

These phenomena can be analyzed in terms of "degrees of freedom." If a clerk tried to write down a relation which was fixed under $\pi$, he would not find (unless $\pi$ were the identity) complete freedom to choose every one of $A$ 's $n^{2}$ elements arbitrarily. The maximum number he might freely so choose may be called the number of $d e$ grees of freedom in a general dyadic relation under $\pi$, and written $d_{2}(\pi)$.

When $A$ is an $m$-adic relation it can be broken up into "blocks" according to the cycle structure of $\pi$ in the same way. Here a block, $A_{0}$, corresponds to a selection of $m$ cycles from $\pi$, allowing repetitions: in $A_{0}$ the first indices will be controlled by a certain $r_{1}$-cycle, second indices by an $r_{2}$-cycle, etc. As in the dyadic case, $t_{\pi}(A)=A$ entails equating of certain of the block's entries; again $\pi$ permits a certain number of degrees of freedom in the general $m$-adic relation, $d_{m}(\pi)$.

2 Numbers in brackets refer to the bibliography at the end of this paper. 
Lemma. The number of degrees of freedom in the block $A_{0}$ under $\pi$ is $(1 / c)\left(r_{1} \cdots r_{m}\right)$, where $c$ is the 1.c.m. of the lengths of the cycles governing the various indices.

Proof. Let $a_{i_{1} \ldots i_{m}}$ be any entry of $A_{0}$, and write out all the formally distinct $r_{1} \cdots r_{m}$ entries:

$$
\left.a_{i_{1}+1, \cdots, i_{m}+1}=\cdots=a_{i_{1}+\left(r_{1}\right.} \cdots r_{m}\right), \ldots, i_{m}+\left(r_{1} \cdots r_{m}\right) .
$$

As before, the $k$ th index is computed modulo $r_{k}$. If the $i$ th and the $j$ th from the left are in fact the same entry, then $i \equiv j\left(\bmod r_{k}\right)$ for all $k$, i.e. $i \equiv j(\bmod c)$ so that the first $c$ entries are certainly distinct. Clearly, however, these are all the distinct ones. If these do not exhaust the block (i.e., if $c \neq r_{1} \cdots r_{m}$ ), choose a new entry and repeat.

3. The main theorem. This result is naturally stated in two parts: the first extends the lemma from one block to the whole matrix, while the second assembles the facts now at hand for the desired formula.

THEOREM 1. If $\pi$ has cycle structure $\left(p_{1}, \cdots, p_{n}\right)$, then

$$
d_{m}(\pi)=\sum_{r_{1}, \cdots, r_{m}=1}^{n}\left(p_{r_{1}} \cdots p_{r_{m}}\right)\left(r_{1} \cdots r_{m}\right) / c
$$

with $c$, as before, the 1.c.m. of the $r_{k}$ 's.

Proof. For each of the $\left(\sum p_{k}\right)^{m}$ ways of selecting $m$ cycles of $\pi$, choose the corresponding block as above. By disjointness, the number of degrees of freedom in each block so singled out is unaffected by that in any of the others. Since there are just $\left(p_{r_{1}} \cdots p_{r_{m}}\right)$ blocks giving the result of the lemma, the conclusion follows.

THEOREM 2. The number of structures of an m-adic relation on a set of $n$ elements is

$$
s t_{m}(n)=\sum_{[\pi]} n(\pi) 2^{d_{m}(x)}
$$

where $d_{m}(\pi)$ is determined as in Theorem 1 for $\pi$ in $\mathfrak{S}_{n}$.

Proof. This is equation (1) with the newly-acquired results giving $f\left(t_{\pi}\right)=2^{d_{m}(\pi)}$.

4. Special kinds of dyadic relations. Equation (1) tells how to count the orbits in any set acted on by $\mathfrak{S}_{n}$. Hence to count the number of (always hereafter dyadic) relations which are nonisomorphic having some particular property, it is again only necessary to count the number with this property fixed under representative $\pi$ 's. 
In each case a reasonable way to start is to apply the reduction of the counting lemma: divide the matrix into submatrix blocks corresponding to pairs of cycles chosen from $\pi$. The degrees of freedom in these blocks may no longer be independent (e.g., when the property is that of symmetry) but for some kinds of relations the nature of the dependence is transparent.

For ready reference, Theorem 1 may be set out explicitly for dyadics

$$
d(\pi)=d_{2}(\pi)=\sum_{h, k=1}^{n} p_{h} p_{k}(h, k)
$$

where $(h, k)$ is the g.c.d. of $h$ and $k$.

TheOREM 3. The number of nonisomorphic reflexive (or irreflexive) relations on a set of $n$ elements is

$$
\begin{aligned}
& \operatorname{ref}(n)=\sum_{[\pi]} n(\pi) 2^{d_{\text {ret }}(\pi)}, \\
& d_{\mathrm{ref}}(\pi)=d(\pi)-\sum_{k=1}^{n} p_{k} .
\end{aligned}
$$

Proof. In a reflexive relation all $a_{i i}=1$ (sometimes called totally reflexive); irreflexive says all $a_{i i}=0$. Now the diagonal elements are always permuted among themselves by every $t_{\pi}$. The number of degrees of freedom in equation (2) which arise on the diagonal is just the number of cycles in $\pi$, that is $\sum p_{k}$.

CoRollary 3.A. The number of directed graphs on nodes $P_{1}, \cdots$, $P_{n}$ with at most one arrow $P_{i} \rightarrow P_{j}$ is simply ref $(n) .^{3}$

For a systematic approach to the succeeding arguments, the blocks of the counting lemma can be classified as diagonal, near-diagonal or off-diagonal according as they (i) contain elements of the main diagonal, (ii) fail to do so, but are nevertheless square submatrices (have their rows and columns permuted by cycles which, though different, are of the same length), or (iii) have different numbers of rows and columns. (The names derive from the canonical way of writing $\pi$.)

THEOREм 4. The number of nonisomorphic symmetric relations on $n$ elements is

\footnotetext{
3 Frank Harary has derived a generating function for directed graphs by an adaptation of Polya's methods [10]; these methods, I learn, rest on the same group theory facts as mine, though they are used for different problems and with a different combinatorial approach.
} 


$$
\begin{aligned}
\operatorname{sym}(n) & =\sum_{[\pi]} n(\pi) 2^{d_{\text {dom }}(\pi)}, \\
d_{\text {aym }}(\pi) & =\sum_{k=1}^{n} p_{k}\left\{\left[\frac{k}{2}\right]+1+k\left(p_{k}-1\right) / 2\right\}+\sum_{k<k} p_{h} p_{k}(h, k) ;
\end{aligned}
$$

$[k / 2]$ is the greatest integer function.

Proof. The off-diagonal and near-diagonal blocks fall into transpose pairs. Symmetry thus makes no change in $d(\pi)$ within each, but since each determines its reflection the $d(\pi)$ for all of these can be determined from half, say those below the diagonal. By (2), the off-diagonal blocks account for the sum on the right. Since for each $k$ there are $p_{k}\left(p_{k}-1\right) / 2$ near diagonals to consider, each with $k$ degrees of freedom, the right-hand part of the first sum is accounted for by the near-diagonal blocks.

A diagonal block has its rows and columns permuted by the same $k$-cycle, say $(12 \cdots k)$. Here there are $k$ strings of equated entries, the broken diagonals:

$$
\begin{aligned}
& S_{0}: a_{22}=a_{33}=\cdots=a_{11}, \\
& \vdots \\
& \dot{S}_{m}: a_{2+m, 2}=a_{3+m, 3}=\cdots=a_{1+m, 1}, \\
& \dot{S_{k-1}}: a_{12}=a_{23}=\cdots=a_{k 1 .} .
\end{aligned}
$$

In general these strings fall into transpose-pairs: where the requirement of symmetry equates two of them, the two degrees of freedom they formerly enjoyed are cut to one. But there are either one or two exceptional strings. If $k$ is odd, $S_{0}$ is its own transpose, while every other $S_{m}$ is paired with $S_{k-m}$. If $k$ is even, though, both $S_{0}$ and $S_{k / 2}$ are exceptional. In either case the $d(\pi)$ in this block is $[k / 2]+1$, and there are $p_{k}$ such diagonal blocks.

CoROLLARY 4.A. The number of nonisomorphic irreflexive and symmetric relations on $n$ elements is

$$
\begin{aligned}
& \operatorname{irs}(n)=\sum_{[\pi]} n(\pi) 2^{d_{i r s}(\pi)}, \\
& d_{\text {irs }}(\pi)=d_{\text {sym }}(\pi)-\sum_{k=1}^{n} p_{k} .
\end{aligned}
$$

COROLLARY 4.B. The number of ordinary graphs on nodes with at most one arc between any two nodes is irs $(n)$.

Like Corollary 3.A, this is only a different formula for a known 
result. Using the powerful methods of his [10], Pólya has derived a generating function whose coefficients give the number of ordinary graphs on $n$ nodes with $k$ arcs, for each $k=0, \cdots, n(n-1) / 2$. As yet unpublished, this result was communicated by Pólya to Riddell, who used it in his thesis [11].

THEOREM 5. The number of nonisomorphic asymmetric (or antisymmetric) relations on $n$ elements is

$$
\begin{aligned}
& \operatorname{asym}(n)=\sum_{[\pi]} n(\pi) 3^{d_{\text {adym }}(\pi)}, \\
& d_{\text {asym }}(\pi)=\sum_{k=1}^{n} p_{k}\left\{\left[\frac{k-1}{2}\right]+k\left(p_{k}-1\right) / 2\right\}+\sum_{h<k} p_{h} p_{k}(h, k) .
\end{aligned}
$$

Proof. In an asymmetric relation $a_{i j}=1$ implies $a_{j i}=0$ while all $a_{i i}=0$ (for anti-symmetric, the same holds except that all $a_{i i}=1$ ). The feature distinguishing this from the symmetric case is that a transpose pair, which there took only the values $(0,0)$ or $(1,1)$, may now be $(0,0),(1,0)$, or $(0,1)$. This accounts for the powers of three in the sum. Within the diagonal blocks, if a string is its own transpose then it must be zero. This cuts down $d(\pi)$ by 2 in the even case, by 1 in the odd, and accounts for the change in the first part of the first sum.

Counting the number of non-isomorphic functional relations introduces further modifications of method. Although equation (1) and the block structure under $\pi$ remain the basic tools, the "degree of freedom" concept can now be discarded.

If $A$ is a function it must have just $n 1$ 's, one in each row. When there is no further restriction on $A$, the $n$ ways of choosing the 1 in any row are independent of those in any other, so that there are $n^{n}$ functions altogether. In general, however, requiring that $t_{\pi}(A)=A$ may lead to equating of two or more entries in some rows: and if $a_{i j}=a_{i k}$ holds of distinct entries in a functional matrix, then neither could be 1 .

Lemma. If $A_{0}$ is a $k \times m$ block under $\pi$ of the functional matrix $A$, then

(i) if $m \nmid k, A_{0}$ consists entirely of zeros;

(ii) if $m \mid k$, then $A_{0}$ may either have $k 1$ 's or none: and there are $m$ different ways in which $k 1$ 's can be chosen in $A_{0}$.

Proof. (i) If $m \nmid k$, then the 1.c.m. $[k, m]>k$, so that if $a_{i j}=1$, then there is a distinct entry in the $i$ th row, viz. $a_{i+k, j+k}$, which is also 1 , contradicting the assumption that $A$ is a function. 
(ii) On the other hand, if $m \mid k$ any one of the $m$ elements of the $i$ th row may be chosen as 1 ; this choice determines all $k 1$ 's of the block.

THEOREM 6. The number of nonisomorphic functions on a set of $n$ elements (to itself) is

$$
\sum_{[\pi]} n(\pi) f_{0}\left(t_{\pi}\right)
$$

where

$$
f_{0}\left(\dot{t_{\pi}}\right)=\prod_{k=1}^{n}\left(\sum_{m \mid k} m p_{m}\right)^{p_{k}}
$$

Proof. The convention that $0^{0}=1$ assures nonzero factors on the right even when $p_{k}=0$; they are certainly nonzero otherwise. The different possible ways of choosing the 1's can be counted in terms of horizontal bands across $A$ corresponding to the cycles of $\pi$. In each of the $p_{k}$ bands corresponding to $k$-cycles in $\pi$ the lemma says there are just $\sum_{m \mid k} m p_{m}$ ways to choose; independence of the choices in different bands determines the formula from this fact.

5. Conclusions. Many kinds of mathematical systems, such as graphs, partially ordered sets, groups, etc., can be completely described in terms of one relation. The methods of this paper might be applied in any such instance to reduce the question of how many "essentially distinct" such objects there are (finite) to a particularly simple combinatorial question. In this way, for example, we have incidentally come on alternatives to two known results of graph theory.

On the other hand, the combinatorial problem which arises in any nontrivial investigation of this sort may be unapproachable. I have tried at some length to discover a way to count the transitive, or transitive and anti-symmetric, relations fixed under arbitrary permutations, without any results. It would seem likely that the particular kind of triadic relation which is a group, for instance, would be even harder to grasp.

There is also a word due about the extent to which Theorem 2 is a solution to the original problem. In theory, of course, one can prescribe a method for writing down all the (additive) partitions of $n$ which define the summation. The theorem thus provides the "general formula stating the number of structures of one dyadic relation for finite $N^{\prime \prime}$ (and more) whose lack Carnap deplored [6, p. 124].

For practical purposes, as a matter of fact, the soon embarrassing 
number of these partitions is the least of the difficulties. For $K$ $=(\log 2)^{-1}$ :

$$
2^{n^{2}-K \log n}<\operatorname{st}_{2}(n)<2^{n^{3}} .
$$

For instance, $\mathrm{st}_{2}(10)>10^{20}$. In practice, the problem will be determined in the case of each of the formulas for various kinds of relations by the question of just how closely the logicians or other investigators would like to have each of the several numbers approximated. The problem of getting good approximations, unlike that of transitivity (which I just could not handle), is one that I have considered outside of the bounds of this paper.

There are also related problems which arise both in Carnap's theory of inductive logic and in the suggested application to the study of finite mathematical systems. The most obvious of these are the determinations of the numbers of "plural" and "mixed" structures: e.g., for pairs of dyadic relations, or for property, dyadic and triadic relation, say. Perhaps farther afield is the question of determining "structure characteristics" $[5$, p. 55] or, in general, that of finding a good set of invariants. While the methods of this paper might be easily extensible to the plural and mixed problems, I do not see that they throw any new light on the latter problem.

Although the very small numbers are likely in many ways to be exceptional, the following facts about dyadic relations with $n \leqq 5$ may be interesting.

\begin{tabular}{ccccccc}
$n$ & st & ref & sym & irs & asym & fcn \\
2 & 10 & 3 & 6 & 2 & 2 & 3 \\
\hline 3 & 104 & 16 & 20 & 4 & 7 & 7 \\
\hline 4 & 3,044 & 218 & 90 & 11 & 42 & 19 \\
\hline 5 & 291,968 & $\begin{array}{c}9,608 \\
\text { BIBLIOGRAPHY }\end{array}$
\end{tabular}

1. J. C. C. McKinsey, Postulates for the calculus of binary relations, J. Symbolic Logic vol. 5 (1940) pp. 85-97.

2. A. Tarski, On the calculus of relations, J. Symbolic Logic vol. 6 (1941) pp. 73-89.

3. A. N. Whitehead and B. Russell, Principia Mathematica, Cambridge, 1910.

4. B. Russell, Introduction to mathematical philosophy, London, 1920.

5. R. Carnap, Abriss der Logistik, Vienna, 1929.

6. - Logical foundations of probability, Chicago, 1950.

7. F. I. Mautner, An extension of Klein's Erlanger program: Logic as invariant theory, Amer. J. Math. vol. 68 (1946) pp. 345-384.

8. N. Bourbaki, Elements de Mathematique: Algebre: chap. i, Structures alg8briques, Paris, 1951. 
9. F. D. Murnaghan, The theory of group representations, Baltimore, 1938.

10. G. P6lya, Kombinatorische Anzahlbestimmungen fïr Gruppen, Graphen und chemische Verbindungen, Acta Math. vol. 68 (1937) pp. 145-254.

11. R. J. Riddell, Contributions to the theory of condensation, University of Michigan Dissertation, 1951.

UNIVERSITY OF MichigaN

\section{TWO NOTES ON RECURSIVELY ENUMERABLE SETS}

\section{J. C. E. DEKKER}

Introduction. These notes are based on E. L. Post's paper Recursively enumerable sets of positive integers and their decision problems ${ }^{1}$ to which we shall refer as RES. The reader is assumed to be familiar with $\$ \S 1-5$ and 9 of this paper. In the first note we shall discuss some algebraic properties of simple and hypersimple sets. In the second note we shall prove the existence of a recursively enumerable set which is neither recursive nor creative nor simple and discuss its degree of unsolvability relative to one-one reducibility and relative to many-one reducibility.

Notations and terminology. A collection of non-negative integers is called a set, a collection of sets is called a class. An empty collection is considered as a special case of a finite collection. Non-negative integers and functions are denoted by small Latin letters, sets by small Greek letters, and classes by capital Latin letters. The Boolean operations are denoted by " + " for addition, " $X$," "." or juxtaposition for multiplication, " sion. Proper inclusion between classes is denoted by " $\mathrm{C}_{+}$."

$\epsilon={ }_{d f}$ the set of all non-negative integers.

$0={ }_{d f}$ the empty set.

$\kappa={ }_{d f}$ the complete set defined on p. 295 of RES.

$\zeta={ }_{d f}$ the simple set defined on p. 298 of RES.

$P={ }_{d f}$ the class of all sets whose complement is finite.

$Q={ }_{d f}$ the class of all finite sets.

$E={ }_{d f}$ the class of all recursive sets.

$D={ }_{d j} E-(P+Q)$.

Presented to the Society, November 29, 1952; received by the editors October 15, 1952.

${ }^{1}$ Bull. Amer. Math. Soc. vol. 50 (1944) pp. 284-316. 